was required to effect cure. In patients who suffered hemorrhages from floating teeth, bone formed and stabilized the teeth and no further hemorrhages occurred. Ethanol sclerotherapy proved curative in mandibular intraosseous AVMs in patients who had additional facial soft-tissue AVMs and intramaxillary AVMs that were cured as well at long-term followup.

Disclosures W. Yakes: None.

\section{E-087 VIRTUAL ANGIOGRAPHIC RECONSTRUCTED PROJECTIONS FROM FOUR-DIMENSIONAL DIGITAL SUBTRACTION ANGIOGRAPHY ACQUISITION, A FEASIBILITY STUDY}

${ }^{1} \mathrm{~L}$ Pung, ${ }^{2} \mathrm{R}$ Darflinger, ${ }^{2} \mathrm{Y} \mathrm{Yu},{ }^{2} \mathrm{M}$ Alexander, ${ }^{2} \mathrm{~A}$ Nicholson, ${ }^{2} \mathrm{~F}$ Settecase, ${ }^{1} \mathrm{~T}$ Moore, ${ }^{2} \mathrm{M}$ Amans, ${ }^{2} \mathrm{~S}$ Hetts, ${ }^{2} \mathrm{D}$ Cooke. ${ }^{1}$ Neurointerventional Radiology, UCSF Medical Center, Hoffman Estates, IL; ${ }^{2}$ Neurointerventional Radiology, UCSF Medical Center, San Francisco, $C A$

10.1136/neurintsurg-2016-012589.159

Introduction/purpose Digital subtraction angiography (DSA) remains the gold standard for the evaluation of extra- and intracranial vascular pathology. Typically, acquisition of multiple angiographic projections is needed to either elongate the vessel(s) of interest or to separate overlapping vessels. Acquiring multiple projections for each selected vessel is costly in terms of time, contrast load, and radiation exposure. Three dimensional digital subtraction angiography (3 DDSA) enabled angiographers to evaluate single vessel injections in multiple projections. Four dimensional digital subtraction angiography (4 DDSA) provides time-resolved 3D acquisition of both the arterial and venous phases of angiography. The aim of this paper is to evaluate the overall quality of processed 4 DDSA to discriminated vascular pathology from normal anatomy.

Materials/methods 3 D DSA acquisition was performed to capture primarily arterial phase $(260 \mathrm{deg}, 1.5 \mathrm{deg} / \mathrm{f}$, rotation duration: $\sim 6 \mathrm{~s}, 172$ projections, $0.36 \mu \mathrm{Gy} /$ projection) or both arterial and venous phases (260 deg, $0.85 \mathrm{deg} / \mathrm{f}$, rotation duration: $\sim 12$ s, 304 projections, $0.36 \mu \mathrm{Gy} /$ projection). Projection images obtained from the rotational acquisition were combined with the constraining 3D-DSA vascular volumes in order to form a time resolved 4 DDSA. Using an edge enhanced reconstruction kernel, the 4 DDSA volume was visualized with either a smooth or sharp image characteristic with a slice matrix of $512 \times 512$. This was then reconstructed into a Virtual Angiography image (Siemens). A secondary reconstruction of the mask phase of each DSA run was also performed to obtain soft tissue and bone anatomical information, from which standard biplane angiographic projections of the 4 DDSA were reconstructed. Windowing, contrast, brightness and opacity levels were adjusted.

Single frame images of processed 4 DDSA acquisitions were then evaluated by our Inerventional Neuroradiology staff, as were the corresponding standard biplane projections. Images were graded on overall quality $(0=$ nondiagnostic, $1=$ poor, 2 = acceptable, $3=$ good) and ability to discriminate pathology from normal vascular anatomy. Results were compared using standard multivariate two-sample t-test.

Results Four internal carotid artery and one vertebral artery injection were evaluated. All vessels had positive findings. Evaluators identified all positive findings on both 4 DDSA and standard DSA images. There was no significant difference in ability to clear the key branch points or vessels between standard and 4 DDSA (3.84 vs. $3.65, \mathrm{p}=0.220)$. The standard DSA image quality was significantly better than 4 DDSA (20.0 vs. 11.2 , p < 0.001) in composite quality scoring.

Conclusions Pathology was clearly delineated from normal vascular anatomy on 4 DDSA. However, the quality of the processed 4 DDSA images remains inferior to standard DSA projections. 4 DDSA image quality may may be improved by optimizing acquisition parameters and injection rates, but this work illustrates significant limitations in the current post-processing algorithm as the source data demonstrates better quality and resolution prior to processing. As the acquisition and post-processing software improve, single injection 4 DDSA offers distinct advantages of decreased study time, contrast dose, and radiation exposure.

Disclosures L. Pung: 5; C; Siemens Medical Solutions. R. Darflinger: None. J. Yu: None. M. Alexander: None. A. Nicholson: None. F. Settecase: None. T. Moore: 5; C; Siemens Medical Solutions. M. Amans: None. S. Hetts: None. D. Cooke: None.

\section{E-088 A FOLLOW-UP TO TRANSRADIAL ACCESS FOR ACUTE INTERVENTIONAL STROKE THERAPY - A FEASIBILITY STUDY}

J Farkas, N Farkas, S Feuerwerker, A Tiwari, D Turkel-Parrella, K Arcot, K Sivakumar. NYU Lutheran Medical Center, Brooklyn, NY

\subsection{6/neurintsurg-2016-012589.160}

Introduction Interventional stroke therapies are quickly becoming the new standard of care for acute large vessel occlusions. Stentrievers and aspiration catheters, while significantly improving recanalization rates, have increased the need for better catheter tracking and stable positioning within the neurovasculature. Difficulties in accessing the target vessel occlusion results in delays and a potential for poor outcome. The main intra-procedural cause for treatment delay and failure-totreat includes unfavorable vascular anatomy. Type III or bovine aortic arch, extreme tortuosity, aortic aneurysms or severe peripheral vascular disease presents challenging obstacles in access. These anatomical abnormalities can make safe and timely transfemoral access difficult, even impossible at times. Alternative access modes, including transradial access, have proven effective in cardiovascular intervention and may provide advantages in circumventing tortuous vascular anatomy. This modality has been adapted for access in stroke intervention; herein, we present additional results from our previous experience with transradial access for endovascular stroke therapy.

Methods A retrospective review of our stroke database was conducted between January 2013 to February 2016 to identify all patients treated endovascularly through transradial access both as first or second intention. The primary outcome of this study measured the success of radial access in ischemic stroke therapy. Secondary outcome evaluated procedural time between cohorts where radial access was primary versus secondary, and procedural adverse events.

Results In total, 34 patients were identified, 17 were treated transradially as primary, 16 as secondary, and 1 was a tertiary attempt; the median age was 86 years [IQR 74-90].

In patients where radial access was first intentions, the median time from puncture to clot engagement was 69 minutes [IQR 28.5$78]$ (mean $=70.7 \pm 52.4)$, with successful revascularization 